UVX 2008 (2009) 21-27

(C) EDP Sciences, 2009

DOI: $10.1051 / \mathrm{uvx} / 2009005$

\title{
X-ray diffraction for material science
}

\author{
E. Collet, M. Buron, H. Cailleau, M. Lorenc, M. Servol, \\ P. Rabiller and B. Toudic \\ Institut de Physique de Rennes, UMR-CNRS 6251, Université de Rennes 1, 35042 Rennes \\ Cedex, France
}

\begin{abstract}
This paper presents different aspects of $\mathrm{x}$-ray diffraction techniques for material science: investigation of symmetry breaking, electron density analysis, diffuse scattering, aperiodic systems and time-resolved experiments.
\end{abstract}

\section{INTRODUCTION}

Understanding function is stringently connected to the knowledge of structure and diffraction experiments have largely contributed to the understanding of matter at atomic scale [1,2]. It spans from simple molecular structure analysis to more complicated description of matter with subatomic resolution, local order or super-space analysis. As materials can transform through application of different perturbations, diffraction gives a global picture of structural changes. Recently pulsed x-ray source made it possible to develop time-resolved $\mathrm{x}$-ray diffraction down to atomic motion time-scales (100 fs).

\section{BASICS OF X-RAY DIFFRACTION}

$\mathrm{X}$-ray scattering pattern provides direct information on the structural organization of matter. In a crystal (Fig. 1), the atoms are arranged in a 3D periodic regular pattern. A small volume element exists (the unit cell), which by periodic repetition in three dimensions describes the crystal. The dimensions of the unit cell are described by three axes: $\mathbf{a}, \mathbf{b}, \mathbf{c}$ and the angles between them $\alpha, \beta, \gamma$. An incident $\mathrm{X}$-ray beam interacts with electrons around atoms, which scatter the incoming X-rays. As atoms in a crystal are arranged in a regular pattern, constructive interferences exist for X-ray beams leaving the sample at specific directions $\mathbf{Q}=\mathbf{k}_{\mathbf{d}}-\mathbf{k}_{\mathbf{i}}$, where $\mathbf{k}_{\mathbf{d}}$ and $\mathbf{k}_{\mathbf{i}}$ are respectively the diffracted and incident wave vector of the X-ray. These directions correspond to the nodes of the reciprocal lattice (defined by $\mathbf{a}^{*}, \mathbf{b}^{*}, \mathbf{c}^{*}$ ). Each of these directions $\mathbf{Q}$ is given by the coordinates $\mathrm{h}, \mathrm{k}$ and $\mathrm{l}$ on the reciprocal lattice $\mathbf{Q}=\mathrm{ha}^{*}+\mathrm{kb} \mathbf{b}^{*}+\mathrm{lc} \mathbf{c}^{*}$.

If one looks at a scattering pattern recorded on two-dimensional (2D) detectors, one observes a number of more or less intense peaks (Fig. 2), as a manifestation of a periodic or quasi-periodic longrange structural order. It means that the average state (position, orientation, configuration...) of one molecule at a given position fully determines that of other equivalent molecules over very long distances through translation symmetry, or more complicated type of ordering. Sometimes additional weaker diffuse intensities exist outside of these peaks.

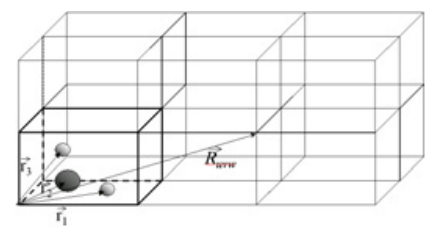

Figure 1. Schematic representation of a three-dimensional periodic crystal. Each unit-cell (dark lines) defined by a lattice parameter set $\mathbf{a}, \mathbf{b}, \mathbf{c}$ contains $\mathrm{N}$ atoms at positions $\mathbf{r}_{\mathbf{j}}$. 

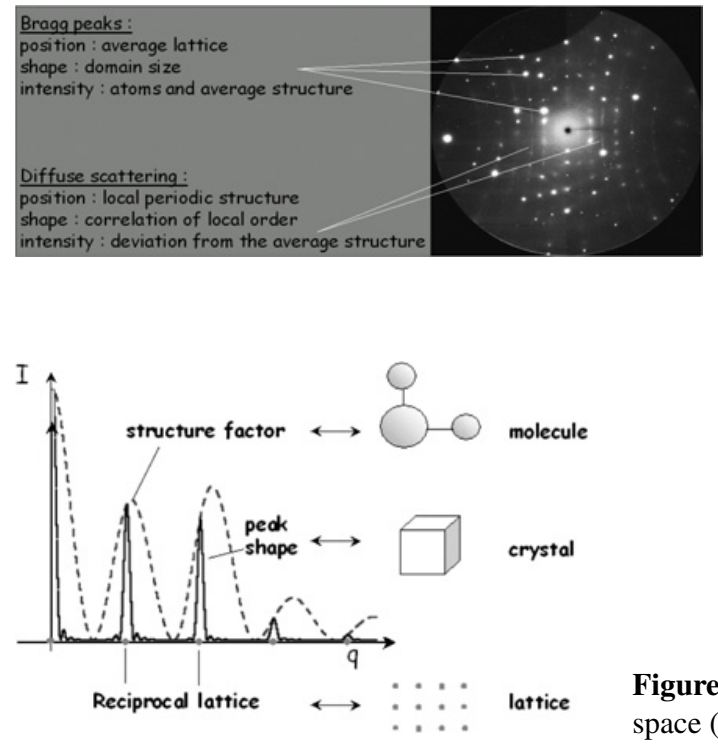

Figure 2. X-ray scattering pattern recorded on a CCD camera in a quasi $1 \mathrm{D}$ system.
Figure 3. Relations between the reciprocal space (left) and real space (right).

The measured intensity in a Bragg peak is proportional to the square modulus of the coherent addition of the amplitudes scattered by individual atoms within the unit cell. The efficiency for each atom is described through the atomic form factor $f_{j}$ (which depends on the chemical nature, i.e. the number of electrons). As atoms occupy different positions within the unit cell, a phase term has to be taken into account, which depends on the direction (h $\mathrm{k} \mathrm{l}$ ). The scattered intensity is given by: $I(h k l)=\left|\Sigma f_{j} \exp \left(2 i \pi\left(h x_{j}+k y_{j}+l z_{j}\right)\right)\right|^{2}$.

These different basic features are illustrated in figure 3 , which refers to a very simple example where every unit cell only contains a small molecule. The position of Bragg peaks is related to the 3D periodic lattice, which allows determining these characteristics (unit cell parameters). Their intensity depends on the atomic structure.

Obtaining a crystalline structure corresponds therefore to the finding of the average coordinates of each atom $\mathrm{j}$ in the unit cell. The number of parameters that may modify intensities corresponds to the number of atoms times the number of degrees of freedom per atoms ( 3 coordinates and anisotropic thermal motion). One can do so using commercial softwares such as SHELX, which use iterative process to refine all parameters of such an atomic model against experimental structure data. Solving a structure of a molecular material requires measuring few thousands Bragg peaks, and even more for biological molecules or accurate electron density distribution.

\section{ELECTRON DENSITY ANALYSIS}

In the above description the atomic scattering power $f_{j}$ is that of atoms where the electron density is spherically distributed around atomic nuclei. Thus the $f_{j}$ 's only depend on $|Q|$. It is well known that atoms form bonds and thus spherical symmetry is almost always broken. At a given position $\mathbf{r}$ from a nucleus and within a tiny volume $d^{3} r$, there are $\mathrm{dn}=\mathrm{nj}(\mathrm{r}) \mathrm{d}^{3} r$ electrons scattering the same way with a phase shift $\exp (-\mathbf{Q} . \mathbf{r})$. The scattering amplitude of an atom $\mathrm{j}$ is then given by the Fourier transform of the atomic electron density $\mathrm{nj}(\mathrm{r})$.

$$
f_{j}(\mathbf{Q})=\int \mathbf{n}(\mathbf{r}) \mathbf{e}^{(-\mathbf{i} \mathbf{Q} \cdot \mathbf{r})} \mathbf{d}^{3} \mathbf{r}
$$




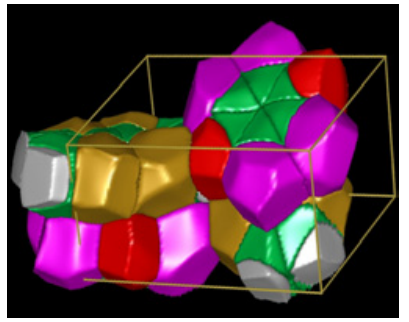

Figure 4. Electron density mapping observed in a molecular crystal. Electron density mapping observed in a molecular crystal.

In the frame of non spherical atoms a symmetry adapted expansion has to be used to describe the electron density. One of the most popular model is due to Hansen and Coppens [3]:

$$
n_{j}(r, \theta, \phi)=n_{\text {core }}(r)+n_{v a l}(\kappa r)+\sum_{l, m} P_{l, m} R_{l}\left(\kappa^{\prime} r\right) . Y_{l, m}(\theta, \phi)
$$

Nowadays high-resolution measurements allow very accurate determination of the electron density, which can be investigated within the topological analysis approach [4]. Qualitative and quantitative atomic and inter-atomic quantities can be derived for a better understanding of chemical bonding. For instance, one can look for atomic basins (Fig. 4), and then count electrons to get a unique definition of intermolecular charge transfer. One can check bondpaths and their bifurcations at phase tansitions [5] or associated with photoinduced switching [6].

\section{PHASE TRANSITION AND SYMMETRY BREAKING}

When material transforms, many phase transitions are associated with symmetry breaking and ordering phenomena, which can be probed by diffraction techniques. Ferroelectric or ferromagnetic phase transitions are well-known examples, but other types of structural and/or electronic order can be probed by diffraction too (charge-ordering ... ). When symmetry breaking occurs, some of the relations between equivalent positions of the crystal are no more satisfied. Symmetry operators in crystals limit the number of degrees of freedom: to each average position of a given atom, a symmetry operator associates an equivalent site $j^{\prime}$. For example, in the case of inversion symmetry to each $\left(x_{j}, y_{j}, z_{j}\right)$ corresponds $\left(-x_{j},-y_{j},-z_{j}\right)$. It is well known from Landau theory of phase transitions [7] that every density of probability $\rho$ (electronic, atomic position, spin, ...) describing the crystalline structure in the two phases can be expressed as $\rho=\rho_{0}+\Delta \rho$ where the first term $\rho_{0}$ is totally symmetric with respect to the highsymmetry phase and the second term $\Delta \rho$ describes the symmetry lowering arising in the low-symmetry phase [7]. This last term is non-zero only in this low-symmetry phase and is proportional to the order parameter associated with the symmetry breaking.

Lets take an example with the partial ordering of spin states in the compound [Fe(2picolylamine)3], $\mathrm{Cl}_{2}, \mathrm{EtOH}$ [8]. This system undergoes a phase transition schematically shown in Fig. 5

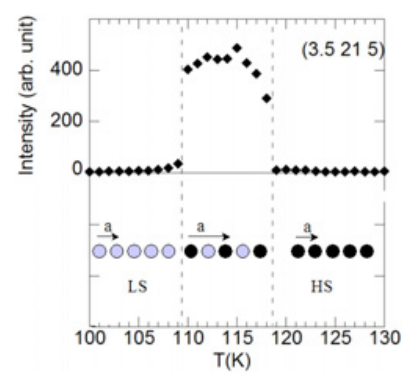

Figure 5. Doubling of the parameter a in the IP phase where HS (black circles) and LS (grey) molecules alternates. This is associated with the appearance of new Bragg peaks in the IP phase. 
between high temperature phase where all molecules are in the high spin (HS, S=2) and low temperature phase where all molecules are in the low spin state $(\mathrm{LS}, \mathrm{S}=0)$ ). In the intermediate phase (IP) a fraction of $50 \%$ of HS and LS molecules is detected by magnetic measurements. X-ray diffraction helped check whether there is a statistical spatial distribution of molecules in HS states or some ordering occurs: a regular chain of molecules in HS state (HS HS HS HS HS) correspond to a translation symmetry a (Fig. 5). It is the same for the completly LS state. However, when a regular ordering of HS and LS molecules occurs (Fig. 5) such as (HS LS HS LS) the translation symmetry changes from a periodic set of HS molecules (LS at low temperature) to a periodic set of HS and LS pair in the IP phase. The lattice vector $\mathbf{a}$ is doubled, the reciprocal vector $\mathbf{a}^{*}$ is divided by 2 and therefore new Bragg peak appear, indexed in the high temperature lattice (h $\mathrm{kl}$ ) with $\mathrm{h}=\mathrm{n}+1 / 2$.

\section{DIFFUSE SCATTERING AND LOCAL ORDER}

Perfect crystals in terms of symmetry do not exist [1]: atoms move and molecular states may fluctuate. An instantaneous picture of a crystal would not show a periodic system, as a result of intrinsic incoherent thermal motions and other dynamic or static disordering processes. Thus the instantaneous structure factor of a unit cell $\mathrm{m}$ is composed of two terms, an average value of the structure factor over the crystal and a fluctuation which depends on $\mathrm{m}: F_{m}=\langle F\rangle+\Delta F_{m}$. X-ray scattering by a crystal can be divided in two terms. One described above and known as "Bragg diffraction" is associated with the average 3D crystalline structure (structure factor $\langle F\rangle$ ). A second one, much lower in intensity, known as "diffuse scattering", and associated with the local deviation $\Delta F$. It is more or less spread out within the reciprocal space, depending on the size of these local deviations. Lets consider the prototype quasi-1D molecular solid TTF-CA $[9,10]$. The crystal structure is made of a mixed-stack sequence of alternating TTF donor (D) and CA acceptor (A) molecules. Co-operative electron transfer can occur along the stack and give rise to the so-called one-dimensional (1D) lattice-relaxed charge-transfer exciton-strings. These nano-scale objects, represented by $\ldots D^{0} A^{0}\left(D^{+} A^{-}\right)\left(D^{+} A^{-}\right)\left(D^{+} A^{-}\right) D^{0} A^{0} \ldots$ are made of train of dimerized I molecules extending along the crystalline stacking axis a. As a consequence of a local deviation $\Delta F$ from $\mathrm{F}$ associated with the formation of the exciton-strings, the diffuse scattering signal arises from diffuse planes in the reciprocal lattice, projected on the detector as lines between the Bragg peaks (Fig. 2) and precisely mapped in Fig. 6 with a four-circles diffractometer.
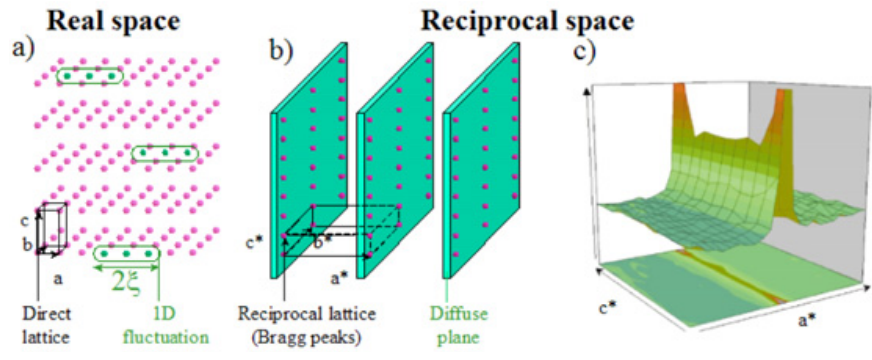

Figure 6. A 3D crystalline lattice (pink spheres, a) diffract $x$-ray at the node of the reciprocal lattice (sphere, b). Local deviations from the average structure factor $\mathrm{F}\left(\Delta F_{m}=F-F_{m}\right.$ blue spheres, a) may appear within a unit cell $\mathrm{n}$. 1D transformations extending over a correlation length $\mathrm{x}$, give rise to diffuse scattering planes (blue, b) passing through some nodes of the reciprocal lattice. c).

\section{APERIODICITY AND SUPER-SPACE}

For some systems in nature, periodicity in a 3D space does not takes place, even though long-range ordering exist. It is the case of quasi-crystals or supramolecular crystals [11]. These last ones are made of diverse array of aperiodic host-guest architectures in which guest molecules are confined with their own periodicity to nanochannels. They assemble in such a way that the ratio of repeat lengths along the channel axis of the host $\left(\mathbf{c}_{\text {host }}\right)$ and guest $\left(\right.$ vecc $\left._{\text {guest }}\right)$ is not a rational number, so the crystal is said to be incommensurate. In the linear tunnels of urea (crystal axis $\mathbf{c}$ ), $\mathbf{c}_{\text {host }}$ and $\mathbf{c}_{\text {guest }}$ are parallel, so one extra 

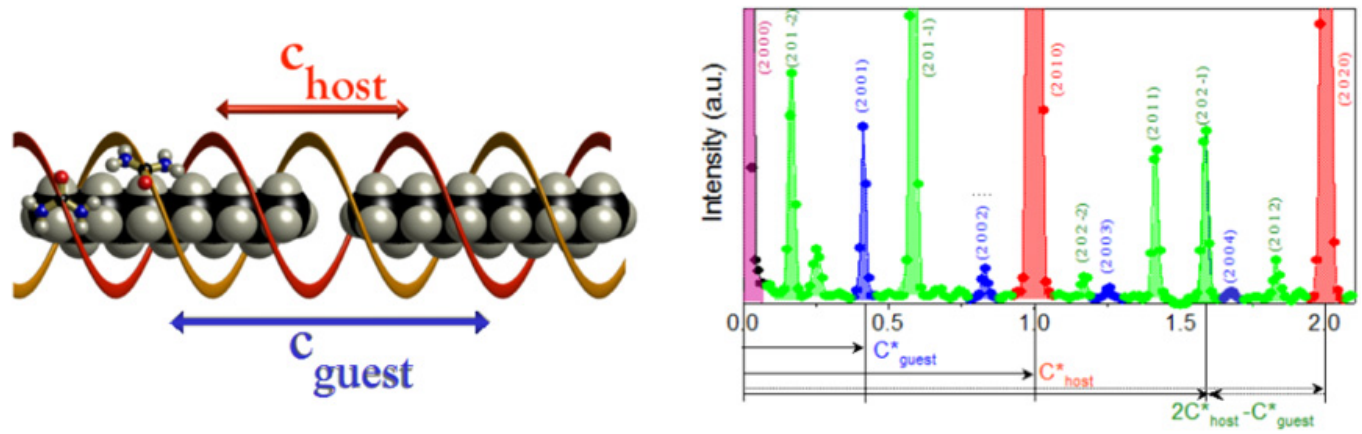

Figure 7. Along the channel, the periodicity of the host urea subsystem is different from the one of the guest. The reciprocal image is characterized by four different types of diffraction peaks (right): the common peaks $(\mathrm{h}, \mathrm{k}, 0,0)$ in purple, the host peaks $(\mathrm{h}, \mathrm{k}, 1,0)$ in red, the guest peaks $(\mathrm{h}, \mathrm{k}, 0, \mathrm{~m})$ in blue and the intermodulation satellite peaks $(\mathrm{h}, \mathrm{k}, \mathrm{l}, \mathrm{m})$ in green, which are the summation positions of $\mathbf{c}_{\text {guest }}^{*}$ and $\mathbf{c}_{\text {host }}^{*}$.

parameter is required to describe the system (Fig. 7). For this nanotubular intergrowth structure, with its single incommensurate direction (c), a four-dimensional superspace description gives the positions of all of the Bragg peaks [12,13]: $\mathbf{Q}_{\text {hklm }}=h \mathbf{a}^{*}+k \mathbf{b}^{*}+l \mathbf{c}_{\text {host }}^{*}+m \mathbf{c}_{\text {guest }}^{*}$, where $\mathbf{a}^{*}, \mathbf{b}^{*}, \mathbf{c}_{\text {host }}^{*}$ and $\mathbf{c}_{\text {guest }}^{*}$ are the reciprocal unit vectors, which differ along $\mathbf{c}^{*}$. The interaction between the substructures requires treating the system as an integral whole [14]. In the case of collinearity of $\mathbf{c}_{\text {guest }}$ and $\mathbf{c}_{\text {host }}$ description is more simple (Fig. 7). The Bragg peaks may be separated into four distinct classes: peaks from the commensurate $\left(\mathbf{a}^{*}, \mathbf{b}^{*}\right)$ plane are indexed (h $\mathrm{k} 00$ ) and are called common Bragg peaks; host peaks reflecting the mean periodicity of the host and indexed (h k 10$)$; guest peaks reflecting the mean periodicity of the guest are indexed (h $\mathrm{k} 0 \mathrm{~m}$ ); and finally, satellite peaks, which characterize the intermodulation of one substructure on the other, are indexed as (h k $1 \mathrm{~m}$ ) with 1 and $\mathrm{m}$ not equal to zero. All four classes of reflections have been reported in the diffraction patterns of alkane/urea inclusion compounds $[14,15]$. The discovery of aperiodic crystals profoundly changed our perception of crystallography. Until there, the translation symmetry seemed to be the required condition to have Bragg peaks, that is Dirac functions in the reciprocal space. Now it is clear that the only requested condition is the existence of long range order, which can be obtained in materials without a simple repetition of a unit cell. In the early eighties, mathematicians and physicists proved that then the translational symmetry which was absent in the 3-dimensional usual space, was recovered defining higher dimensional spaces. The superspace crystallography generalizes and contains the usual 3D crystallography. In this superspace atomic coordinates are no more described with (xj, yj, zj) but with atomic surface. The increase of the dimensionality allows new structural and dynamical disorder. Theses supplementary degrees of freedom drastically increase the number of structural solutions offered to the materials [16], and also generates specific disorder.

\section{TIME-RESOLVED X-RAY DIFFRACTION: TRACKING ATOMIC MOTION AND MOLECULAR RELAXATION}

Photoinduced phase transitions [17] represent challenging issues for the scientific and technological purpose to control by a laser pulse the co-operative switching of the macroscopic physical state of a material. Probing the dynamical evolution of materials is now possible with pulsed x-ray sources such as synchrotrons or 100 fs laser-based X-ray sources [18-25]. By extending the measurements presented here in the time domain one can directly observe such new out-of-equilibrium processes. Two limit cases may be considered: delocalised electronic excitations giving rise to a collective atomic motion or localised ones at the molecular level. 
The first case corresponds to the fascinating possibility of exciting coherent phonons with a laser pulse. The coherent phonons give rise to a periodic oscillation of the Bragg peak intensity of, since the structure factor itself depends on time as:

$$
I(h k l, t)=\left|\Sigma f_{j} \exp \left(2 i \pi\left(h x_{j}(t)+k y_{j}(t)+l z_{j}(t)\right)\right)\right|^{2}
$$

The ultra-fast X-ray scattering is particularly well adapted to investigate coherent phonons since not only the period but also the polarization (normal coordinate) of the vibration mode can be measured by the analysis of the oscillation amplitude on different Bragg peaks.

The second case will generate local modification of the structure factor as photo-excited molecules structurally relax. As explained above, the coexistence of stable and photoinduced states in the case of a random homogeneous distribution of local photoinduced states is described by an average structure factor $\langle F\rangle$. This factor is a weighted contribution of the photoinduced entity ( $F_{\text {photo }}$, concentration $\mathrm{x}$ ) and the ground-state $\left(F_{\text {stable }}\right.$, concentration $\left.1-\mathrm{x}\right)$ :

$$
\langle F(t)\rangle=x F_{\text {photo }}+(1-x) F_{\text {stable }}
$$

Therefore, as the system evolves with time (proliferation or relaxation of the excited state) the time dependence of the average structure factor $\langle F(t)\rangle$ may be related to the evolution of the excited fraction $\mathrm{x}(\mathrm{t})$ rather than the intra-molecular dynamics of the photoexcited state $F_{\text {photo }}(t)$. Note that intra-molecular dynamics is of the order of optical phonons (100 fs or less), whereas lifetime of photo-excited states may vary from ps to days. The investigation of both contributions requires adequate time-resolutions.

\section{References}

[1] J. Baruchel, J.L. Hodeau, M.S. Lehmann, J.R. Regnard and C.Schlenker , Neutron and Synchrotron Radiation for Condensed Matter Studies, Volume 1 and 2 (Springer-Verlag, Berlin, Editions de Physique, Les Ulis 1993)

[2] J. Als-Nielsen, D. Mc Morrow Elements of Modern X-ray Physics (J. Wiley and soons, Berlin, 2000)

[3] T. Hansen, P. Coppens (1978) Acta Crystallographica A34, 909-921

[4] R. W. F. Bader, Atoms in Molecules: A Quantum Theory, International Series of Monographs on Chemistry 22,. Clarendon Press, Oxford (1990).

[5] V. Oison et al, Journal of Physical Chemistry A, vol. 108, p. 11049-11055, 2004

[6] V. Legrand et al, J. Am. Chem. Soc.,128, (2006) 13921

[7] E. M. Lifshitz and L. P. Pitaevskii, Landau and Lifshitz Course of Theoretical Physics, Volume 5 Statistical physics Part 1 (Pergamon Press, Oxford, 1980).

[8] N. Huby et al, Physical Review B 69, (2004) 020101(R)

[9] E. Collet et al, Europhys. Lett. 57, (2002) 67.

[10] M. Buron-Le Cointe et al, Physical Review Letters 96, (2006) 205503

[11] T. Janssen, G. Chapuis, M. de Boissieu, Aperiodic Crystals: From Modulated Phases to Quasicrystals (Oxford Univ. Press, Oxford, 2007).

[12] J. Marti-Rujas et al, J. Am. Chem. Soc. 126, (2004) 11124.

[13] T. Janssen, A. Janner, A. Looijenga-Vos, P. M. de Wolf, International Tables for Crystallography Vol. C, A. J. C. Wilson, Ed. (Kluwer Academic Publishers, Dordrecht 1995), pp. 797-844.

[14] R. Lefort et al, Phys. Rev. Lett. 77, (1996) 4027-4030.

[15] T. Weber et al, Zeit. für Kristall. 211, (1996) 238.

[16] B. Toudic et al, Science 316, (2008) 69.

[17] S. Koshihara, M. Kuwata-Gonokami J. Phys. Soc. Jpn. 75, (2006) 011001-011008.

[18] E. Collet et al, Science 300, (2003) 612.

[19] F. Schotte et al, Science 300, (2003) 1944. 
[20] R. W. Schoenlein et al, Science 287, (2000) 2237.

[21] A. Rousse et al, Nature 410, (2001) 65.

[22] K. Sokolowski-Tinten et al, Nature 422, 287 (2003).

[23] D. Fritz et al, Science 315, (2007) 633.

[24] A.Cavalleri et al, Nature 442, 05041 (2006).

[25] S. Nozawa et al, J. Synchrotron Rad. 14, (2007) 313. 